\title{
Chronic vagal nerve stimulation exerts additional beneficial effects on the beta-blocker-treated failing heart
}

\author{
Meihua $\mathrm{Li}^{1} \cdot$ Can Zheng ${ }^{1} \cdot$ Toru Kawada $^{1} \cdot$ Masashi Inagaki $^{1} \cdot$ Kazunori Uemura $^{1} \cdot$ Masaru Sugimachi $^{1}$
}

Received: 23 August 2018 / Accepted: 29 October 2018 / Published online: 9 November 2018

(C) The Physiological Society of Japan and Springer Japan KK, part of Springer Nature 2018

\begin{abstract}
Vagal nerve stimulation (VNS) induces bradycardia in chronic heart failure (CHF). We hypothesized that beta-blocker would cover the beneficial effects of VNS on CHF if the anti-beta-adrenergic effect was the main VNS effect. This study investigated the effects of VNS on cardiac remodeling in rats with CHF treated with metoprolol. Two weeks after myocardial infarction, surviving rats were randomly assigned to groups of sham stimulation (SS), sham stimulation with metoprolol (SSM), or VNS with metoprolol (VSM). Compared to the SS group, heart rate was significantly reduced in the SSM and VSM groups. Hemodynamic assessments showed that VSM rats maintained better cardiac pump function and presented higher cardiac index and lower heart weight than SSM rats. VSM was also associated with lower plasma brain natriuretic peptide and norepinephrine levels than SSM. VSM but not SSM improved the 50-day survival rate compared with the SS group. The results suggest that VNS may exert its beneficial effects on the failing heart independently of its anti-beta-adrenergic mechanism.
\end{abstract}

Keywords Vagal nerve stimulation · Beta-blocker $\cdot$ Myocardial infarction $\cdot$ Cardiac remodeling $\cdot$ Chronic heart failure

\section{Introduction}

Left ventricular remodeling after acute myocardial infarction (MI) plays a crucial role in progressive left ventricular dysfunction and subsequent heart failure [1]. The degree of ventricular enlargement is an important predictor of survival in patients with coronary artery disease and chronic heart failure (CHF) [2, 3]. Left ventricular systolic dysfunction is associated with activation of neurohumoral compensatory mechanisms such as sympathetic and renin-angiotensin-aldosterone systems [4]. Chronic activation of these mechanisms exerts deleterious hemodynamic and direct cardiotoxic effects and contributes to progressive deterioration of ventricular function. Conversely, attenuation of these mechanisms improves the survival of patients with CHF. This has been demonstrated for angiotensin-convertingenzyme inhibitors (ACEI), angiotensin II receptor blockers (ARB), and beta-blockers [5, 6]. Despite the development of pharmacological therapy and devices, the overall prognosis

Can Zheng

zhengcan@ncvc.go.jp

1 Department of Cardiovascular Dynamics, National Cerebral and Cardiovascular Center, 5-7-1 Fujishirodai, Suita, Osaka 565-8565, Japan of patients with CHF remains poor, and new therapeutic strategies are necessary. In contrast to sympathetic activation, clinical evidence from the Autonomic Tone and Reflexes After Myocardial Infarction [7] and Cardiac Insufficiency Bisoprolol Study II [8] indicated that diminished cardiac vagal activity and increased heart rate (HR) predict a high mortality rate among patients with CHF. This naturally raised a question of whether augmentation of vagal tone would be a new target for the treatment of CHF. We firstly demonstrated that electrical vagal nerve stimulation (VNS) significantly improves the long-term survival of rats with CHF by preventing the progression of pump failure and cardiac remodeling [9]. Several clinical trials of chronic VNS treatment for patients with CHF have been evaluated and reported the safety and improvement of life quality [10-12]. By contrast, improvements in the efficacy endpoints were unconfirmed in the population with severe CHF. Because these studies were conducted in a different population with various strong pharmaceutical therapeutic backgrounds with inconsistent protocols for VNS, the dissimilar conclusion contrasting with animal studies is not surprising. Knowledge on widely acceptable mechanisms for VNS treatment is still lacking. Therefore, clarification of the mechanisms for VNS-mediated beneficial effects remains important for successful clinical translation. HR reduction by vagal efferent 
activation may partly account for the beneficial effects [13]. However, beta-blocker is the most used drug in the abovementioned trials. If VNS treatment exerts benefits mainly through its anti-beta-adrenergic action, beta-blockade should cover these effects. Clinical study is difficult to verify this hypothesis. There is no literature but a conference report concerning combination therapy of beta-blocker with VNS in an animal study [14]. Therefore, we hypothesized that VNS exerts the beneficial effects via mechanisms beyond its anti-beta-adrenergic action in CHF rats. In this study, we aimed to investigate the effects of chronic VNS on cardiac remodeling and dysfunction in rats with MI treated with metoprolol.

\section{Materials and methods}

\section{Experimental chronic heart failure model}

The care of animals and all animal experiments were performed in strict accordance with the Guide for the Care and Use of Laboratory Animals published by the US National Institutes of Health (NIH Publication No. 85-23, revised 1996), and the Guiding Principles for the Care and Use of Animals in the Field of Physiological Sciences, which have been approved by the Physiological Society of Japan. All protocols were reviewed and approved by the Animal
Subject Committee in the National Cerebral and Cardiovascular Center.

Male Sprague-Dawley rats $(n=100$, body weight: 250-280 g; SLC, Hamamatsu, Japan) were anesthetized with halothane (3\% at induction and $1.2 \%$ during surgery) inhalation, and extensive MI was induced by proximal left coronary artery ligation $[9,15]$. Mortality in animals with MI was 54\% $(n=54)$ within the first $24 \mathrm{~h}$. This high mortality was related to the extensive MI size. We confirmed the infarct size by postmortem examination.

\section{Fabrication of the electrode}

We used the same electrode as in our previous studies [9, 16]. The neck is one of the most active body parts in rats. To increase the flexibility and endurance of the lead, we designed a coil structure using a thin stainless wire (OD $0.03 \mathrm{~mm}$, coated with polyurethane; Unique Medical Co., Ltd., Tokyo, Japan). A segment (4 $\mathrm{mm}$ ) of a commercially available silicone tube (OD $1 \mathrm{~mm}$, ID $0.5 \mathrm{~mm}$ ) was used as a cuff around the nerve. A longitudinal slit was made on the tube, through which the nerve was placed. The wires were penetrated through the thin wall and bent against the cuff, parallel to the cuff's long axis. The other ends of the leads were welded (KTH-MWS, Kondo Technology, Inc., Tokyo, Japan) to the output lead of the generator (Fig. 1a, b).
Fig. 1 Design of the silicone tube-based cuff bipolar electrodes and the hemodynamic responses to right cervical vagal nerve stimulation (VNS) in free-moving rats. a Schematic representation of the structure of the cuff electrode. b Image of the implanted electrode with the cervical vagal nerve. The sample electrode was removed 3 months after the implantation surgery. c Representative recording of $\mathrm{HR}$ and $\mathrm{AP}$ response to VNS in a conscious rat with $\mathrm{CHF}$ (stimulation intensity: current, $0.1 \mathrm{~mA}$; pulse, $0.2 \mathrm{~ms}$; frequency, $20 \mathrm{~Hz}$ ). This panel shows the VNS treatment protocol after the adjustment of the stimulation intensity, which was $0.1 \mathrm{~mA}$. In c, black boxes indicate VNS a

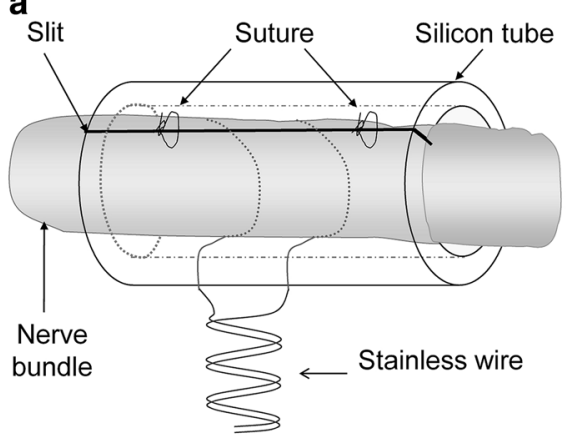

b

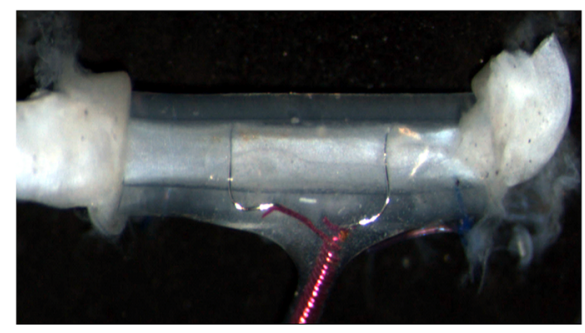

C
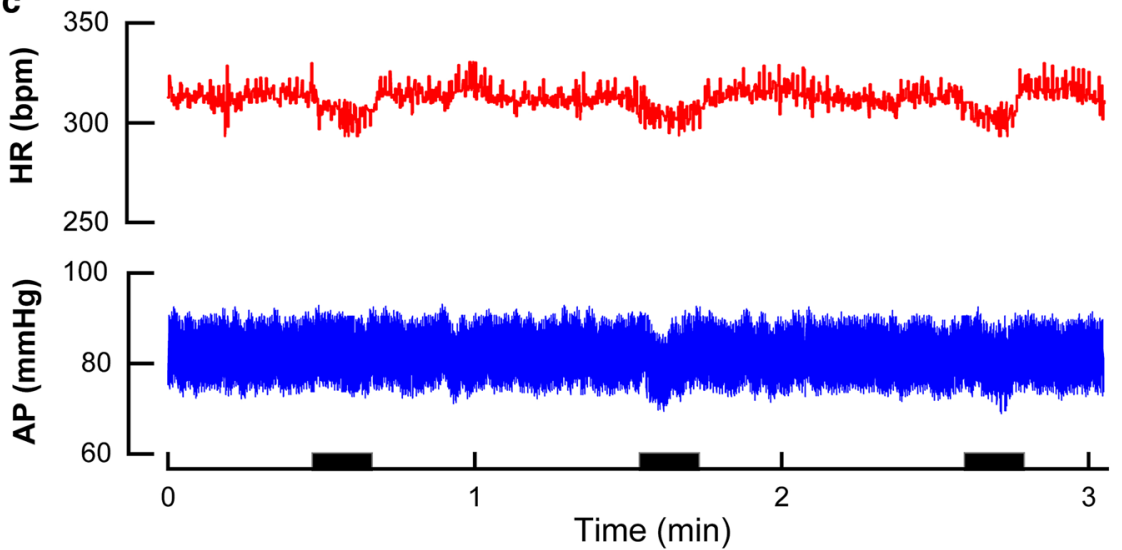


\section{Implantable remote-controlled pulse generator}

As described previously [9], we selected an implantable, real-time remote-controlled system, which consisted of an implantable pulse generator (ISE1000SA; Unimec Inc., Tokyo, Japan) and a command transmission board (ISE1010C; Unimec Inc., Japan). The pulse generator weighed $8 \mathrm{~g}$ with a total volume of $5 \mathrm{ml}$. The working output current range was $0.1-1.0 \mathrm{~mA}$, and the working space of the transmission board was suited for the standard cage ( $L 37.0 \mathrm{~cm}, W 28.5 \mathrm{~cm}$, and $H 9.3 \mathrm{~cm}$ ). We previously tested a hemodynamic response to VNS in conscious CHF rats (Fig. 1c). We regulated the intensity by pulse current $(0.1-0.13 \mathrm{~mA})$ to a submaximal level at which the animals did not show stress-like behavior when the stimulation was conducted. In this manner, VNS did not affect food or water consumption with normal growth rate. During VNS, usually but not always, HR decreased by $20-30$ bpm without great changes of the arterial pressure (AP) in rats with CHF.

\section{Experimental protocols}

One week after inducing MI, survivors were randomly assigned to sham stimulation without metoprolol (SS, $n=18$ ), sham stimulation with metoprolol (SSM, $n=15$ ), or VNS with metoprolol group (VSM, $n=13$ ). The rats in the VSM group were implanted with the above-described electrode around the right cervical vagus and the pulse generator in the back. Similarly, the rats in the SS or SSM group were implanted with a dummy. At the same time, to evaluate the long-term effects of metoprolol with or without VNS on hemodynamics under non-stressful conditions, we implanted the 46 rats with a blood pressure transmitter (TA11PA-C40; DSI, St. Paul, MN, USA). The Teflon tube of the transmitter was inserted into the abdominal aorta to record blood pressure and HR in real time. The recording was sampled at $500 \mathrm{~Hz}$. After another week recovery, we started VNS and selected a pulse frequency of $20 \mathrm{~Hz}$, a pulse width of $0.2 \mathrm{~ms}$, using a duty cycle of $16.7 \%$ (10-s on/50-s off, Fig. 1c) for $24 \mathrm{~h}$ and continued VNS for 6 weeks (limited by battery life of the generator). The effectiveness of VNS was checked weekly by the behavioral and hemodynamic responses in each rat. Metoprolol (Sigma-Aldrich, St. Louis, MO, USA) was added to drinking water $(0.7 \mathrm{~g} / \mathrm{l}$, average dose $70 \mathrm{mg} / \mathrm{kg} /$ day) in the SSM and VSM groups, but not in the SS group. The metoprolol dose was selected to decrease the HR by 20-30 bpm in rats with MI without significant influence on normal growth, according to the findings in a preliminary study. Metoprolol treatment continued until the end of study (7 weeks). At the end of the 6-week stimulation and observation period, the animals were anesthetized with halothane (3\% for induction, $1 \%$ for 5 -min blood sampling operation). Under anesthesia, $3 \mathrm{ml}$ of blood was quickly sampled from the cervical vein for neurohumoral assays. Plasma catecholamine concentrations were measured by high-performance liquid chromatography with electrochemical detection after alumina adsorption. The plasma level of brain natriuretic peptide (BNP) was determined using enzyme-linked immunosorbent assay (BNP-32 Enzyme Immunoassay Kit; Peninsula Laboratory, Inc. Ann Arbor, MI, USA).

\section{Cardiac remodeling and dysfunction study}

To evaluate the effect of VNS on cardiac remodeling, after 1 -week recovery following the last blood sampling, we measured hemodynamics in anesthetized rats with CHF (SS, SSM, and VSM). The anesthesia was maintained using $1.2 \%$ halothane during surgical procedures and $0.6 \%$ halothane during data recording. Left ventricular pressure, AP, and right atrial pressure (RAP) were measured with a 2-Fr catheter-tip micromanometer (SPC-320; Millar Instruments, Inc., Houston, TX, USA) and aortic flow with a transonic flow probe (T-206 flow probe \#2.5 ss66; Transonic Systems Inc., Ithaca, NY, USA), as described previously [9, 15, 17]. All signals were digitized at a rate of $500 \mathrm{~Hz}$ for $1-2 \mathrm{~min}$.

\section{Determination of infarct size and organ weights}

After hemodynamic measurements, the lung and liver were immediately excised and weighed; the heart was excised for subsequent determination of infarct size. As described in previous studies $[9,15,17]$, the biventricles were dissected, weighed, cut from the apex to the base into three transverse slices, and fixed in $4 \%$ phosphate-buffered paraformaldehyde solution. Four-micrometer-thick sections were cut and stained using the Masson trichrome method. Histological images were digitized through a frame grabber and analyzed. Infarct size was calculated from the three slices by dividing the sum of the endocardial lengths of infarcted regions by the sum of the total endocardial circumferences.

\section{Prognosis study}

To examine the outcome of VNS- and metoprolol-treated rats with CHF, we observed a 50-day survival rate in overall treatment time. The rats were inspected daily, and gross postmortem examination was conducted on the dead rats. The heart was removed for subsequent measurements of heart weight and infarct size.

\section{Statistical analysis}

All data were presented as mean and SE values. Data of long-term recorded HR and mean blood pressure (MBP) before and during treatment in each group were examined using one-way analysis of variance (ANOVA) with repeated 
measurements, followed by post hoc Dunnett's test. Differences among the three groups were examined with one-way ANOVA, followed by post hoc Dunnett's test. For hemodynamic and remodeling study data, differences among the three groups were tested by ANOVA, with Scheffé's multiple comparison test. For neurohumoral data, differences among the three groups were tested by ANOVA, followed by post hoc Student-Newman-Keuls test. Survival data are presented as Kaplan-Meier curves, and the effect of treatment on a 50-day survival was analyzed using a log-rank test. For all statistical analyses, the difference was considered significant when $P<0.05$.

\section{Results}

\section{Telemetric hemodynamic measurements in conscious rats with $\mathrm{CHF}$}

The SSM $(n=11)$ and VSM $(n=12)$ groups showed a significant reduction in average HR from the first week compared with the untreated SS group $(n=11)$ and maintained a low level in the following weeks (Fig. 2a). The difference in HR between the SSM and SS groups or between the VSM and SS groups reached more than $40 \mathrm{bpm}$ at the end of the 6-week treatment. However, significant difference was not noted between the VSM and SSM groups. Although no statistically significant differences were found, MBP tended to be higher in the VSM than in the SSM and SS groups (Fig. 2b).

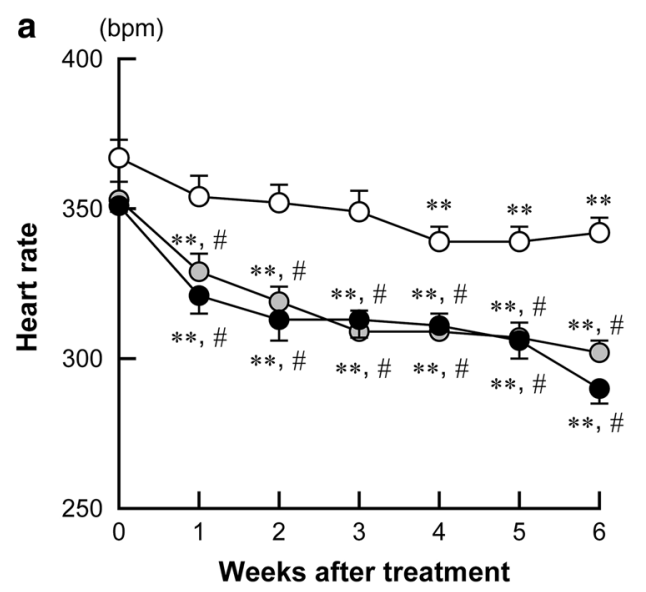

Fig. 2 Effects of a 6-week treatment with sham stimulation (SS, white circles, $n=11$ ), sham stimulation plus metoprolol (SSM, gray circles, $n=11$ ), and vagal stimulation plus metoprolol (VSM, black circles, $n=12$ ) on telemetry hemodynamics in conscious rats with

\section{Hemodynamics and cardiac remodeling}

Acute hemodynamics and cardiac remodeling parameters were measured in anesthetized rats with $\mathrm{CHF}$ at the end of the 7-week treatment (Fig. 3). Although no differences were found in MBP and HR among the three groups (Fig. 3a, b), VSM-treated rats with CHF showed significantly higher cardiac index (Fig. 3c), higher maximum left ventricular pressure $\mathrm{d} P / \mathrm{d} t$ (Fig. 3d), and lower left ventricular end-diastolic pressure (Fig. 3e), and RAP (Fig. 3f) than the SS and SSMtreated rats with CHF. Further improvement of cardiac function in VSM rats was accompanied by a significant decrease in normalized biventricular weight (Table 1). However, significant differences were not noted in body weight, infarct size, or normalized lung and liver weights among the SS, SSM, and VSM-treated rats with CHF (Table 1).

\section{Analysis of plasma neurohumoral levels and prognosis after treatment}

The VSM group showed a lower plasma norepinephrine level than the SS and SSM groups (Fig. 4a). Meanwhile, the SSM and VSM groups had lower plasma epinephrine than the SS group (Fig. 4b). Although the SSM and VSM groups had a lower concentration of plasma BNP than the SS group, the VSM group showed a further decrease in the BNP level than the SSM group (Fig. 4c). During the 7-week observation period, VSM therapy significantly suppressed the mortality rate of rats with CHF (Fig. 5). The number of deaths in the VSM group was only one, which was significantly lower than that in the SS group (Table 1).

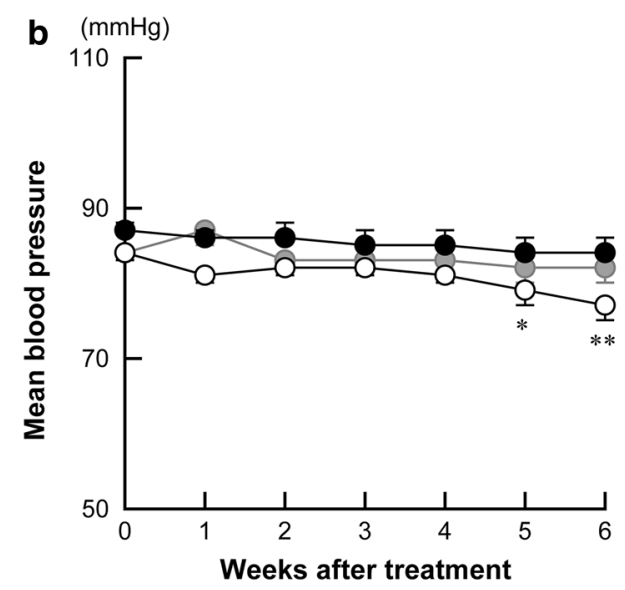

CHF. Each point presented the weekly averaged heart rate (a) and the weekly averaged mean blood pressure (b) of rats with CHF. Data are expressed as mean \pm SEM. $* P<0.05$; $* * P<0.01$ vs. the pre-treatment value of each group (week 0 ); ${ }^{\#} P<0.01$ vs. SS group 
Fig. 3 Effects of treatment with SS (white bars, $n=11$ ), SSM (gray bars, $n=11$ ), and VSM (black bars, $n=12$ ) on a MBP, mean blood pressure; $\mathbf{b} \mathrm{HR}$, heart rate; $\mathbf{c}$ cardiac index; $\mathbf{d}$ $\mathrm{LV}+\mathrm{d} P / \mathrm{d} t_{\max }$, maximum $\mathrm{d} P /$ $\mathrm{d} t$ of left-ventricular pressure; e LVEDP, left ventricular end-diastolic pressure; and $\mathbf{f}$ $\mathrm{RAP}$, right atrial pressure in anesthetized rats with $\mathrm{CHF}$ (chronic heart failure). The assessment was made at the end of a 7-week treatment (1-week recovery after blood sampling). $* P<0.05$ vs. SS group; ${ }^{\#} P<0.05$ vs. SSM group a
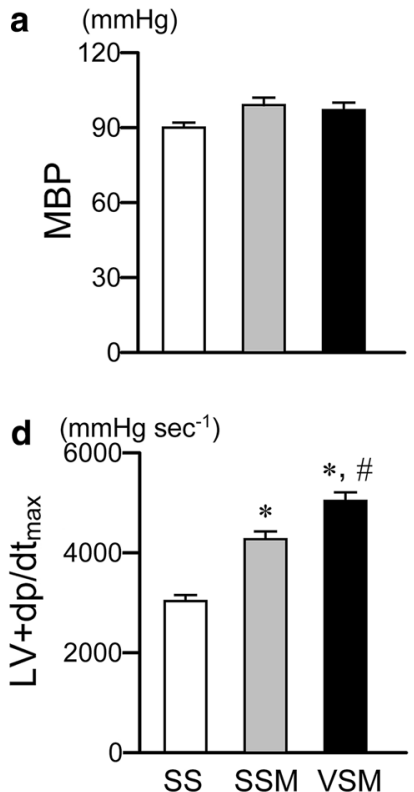
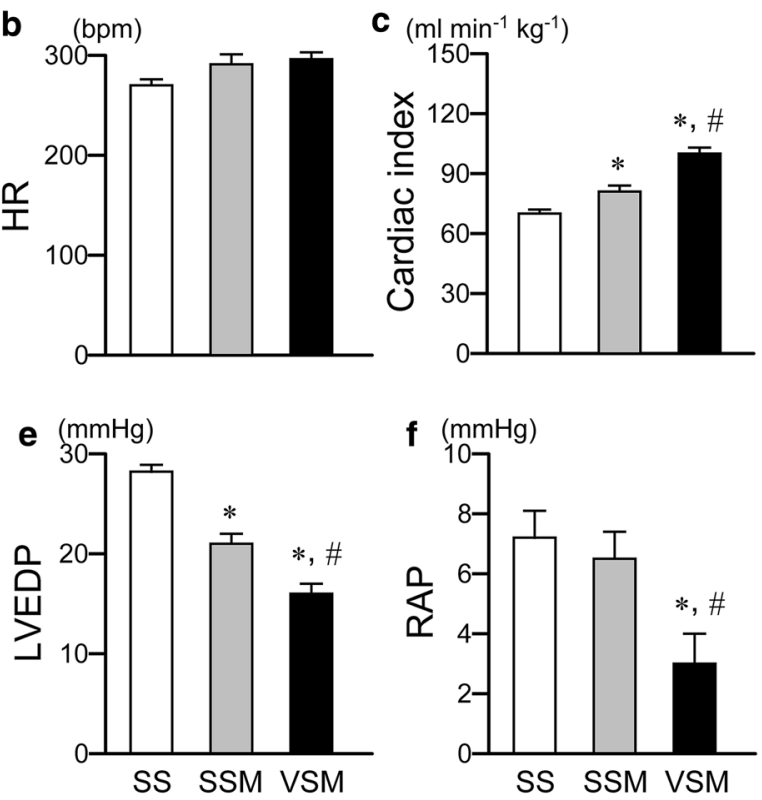

Table 1 The characteristics after 7-week treatment in rats with chronic heart failure

\begin{tabular}{llll}
\hline & SS group $(n=11)$ & SSM group $(n=11)$ & VSM group $(n=12)$ \\
\hline BW $(\mathrm{g})$ & $432 \pm 14$ & $427 \pm 16$ & $425 \pm 8$ \\
Infarct size $(\%)$ & $44 \pm 2$ & $45 \pm 2$ & $44 \pm 1$ \\
HW $(\mathrm{g} / \mathrm{kg})$ & $3.26 \pm 0.19$ & $2.96 \pm 0.06$ & $2.67 \pm 0.08^{*}, \#$ \\
Lung W $(\mathrm{g} / \mathrm{kg})$ & $7.51 \pm 0.45$ & $9.49 \pm 0.83$ & $7.46 \pm 0.85$ \\
Liver W $(\mathrm{g} / \mathrm{kg})$ & $29.38 \pm 0.90$ & $33.49 \pm 1.33$ & $31.14 \pm 0.80$ \\
Death number & $7(7 / 18,39 \%)$ & $4(4 / 15,27 \%)$ & $1(1 / 13,8 \%)^{*}$ \\
\hline
\end{tabular}

Values are mean \pm SEM. For data of BW, HW, lung W, liver $\mathrm{W}$ and infarct size, differences among three groups were tested by ANOVA, with Scheffé's multiple comparison test. The effect of treatment on 50-day survival was analyzed by a log-rank test

$B W$ body weight, $H W$ biventricular weight normalized by body weight, lung $W$ lung weight normalized by body weight, liver $W$ liver weight normalized by body weight

${ }^{*} P<0.05$ vs. SS group

${ }^{\#} P<0.05$ vs. SSM group
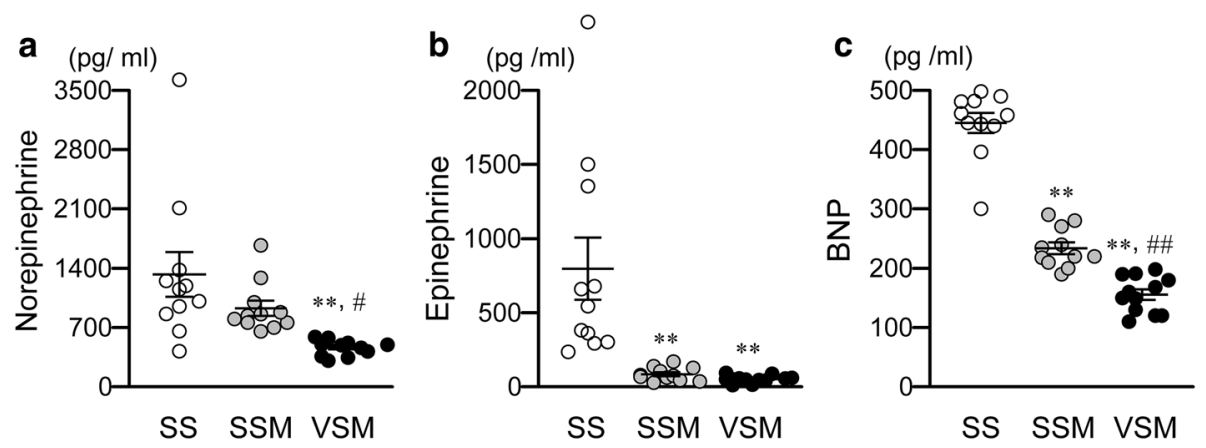

Fig. 4 Comparison of plasma neurohumoral levels after a 6-week treatment with SS (white circles, $n=11$ ), SSM (gray circles, $n=11$ ), and VSM (black circles, $n=12$ ) in rats with chronic heart failure. a
Plasma levels of norepinephrine. b Plasma levels of epinephrine. c Plasma levels of brain natriuretic peptide (BNP). $* * P<0.01$ vs. SS group; ${ }^{\#} P<0.05 ;{ }^{\# \#} P<0.01$ vs. SSM group 


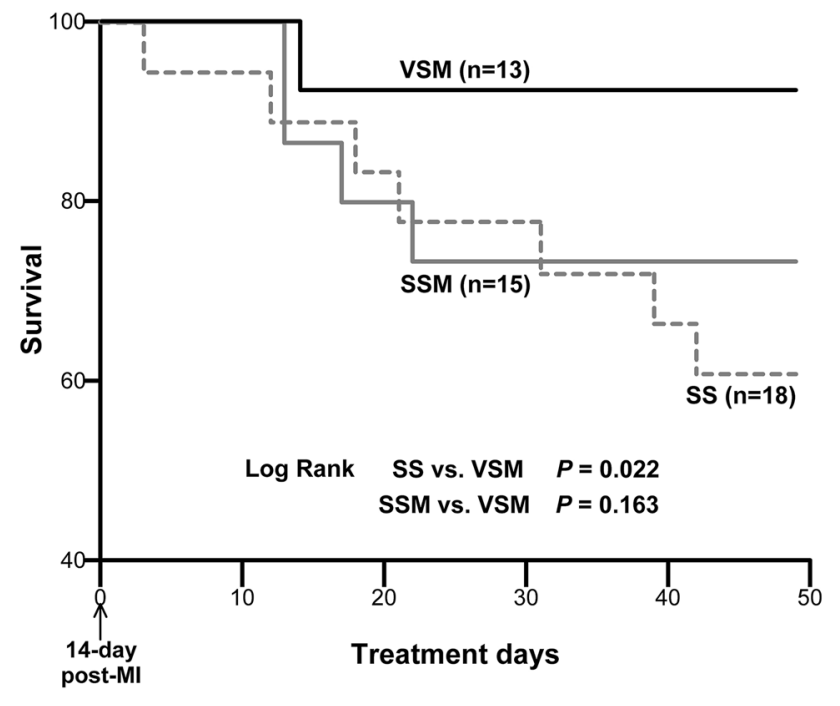

Fig. 5 Effects of treatment with SS (gray dotted line, $n=18$ ), SSM (gray solid line, $n=15$ ), and VSM (black solid line, $n=13$ ) on the survival curves of rats with chronic heart failure. Treatment started 14 days after myocardial infarction (MI) induced by coronary artery ligation. Compared with the SS group, VSM significantly $(P=0.022)$ improved the survival rate for a 50-day observation. No significant difference was found between the VSM and SSM groups

\section{Discussion}

The major results of the present study are as follows. SSM reduced HR, ameliorated cardiac dysfunction, improved cardiac index, and decreased plasma neurohumoral levels, although no difference in normalized heart weight was found compared with SS. On the other hand, VSM further prevented the progression of cardiac remodeling and dysfunction and effectively suppressed mortality and plasma catecholamine and BNP, compared with SSM. These results suggest that VNS exerts its cardioprotective effects on the failing heart independently of its anti-beta-adrenergic mechanism.

\section{Effects of beta-blockade and VNS treatment on CHF}

The present study evaluated the long-term hemodynamics in rats with CHF by using telemetry. Both SSM and VSM therapies similarly reduced HR compared with the SS. The extent of HR reduction was also similar to our previous report that examined the effect of VNS without beta-blockade in rats with CHF [9]. These results imply that chronic VNS does not induce an additional bradycardic effect beyond the bradycardic effect attained by beta-blockade. Hence, chronic VNS may contribute to the reduction of averaged HR mainly through decreasing or inhibiting cardiac sympathetic activity. HR has been proven to be the best predictor for prognosis after MI in patients with congestive heart failure [18]. By inducing bradycardia, beta-blockers have become very important in treating patients with CHF. Considerable evidence is available showing that beta-blockade reduces mortality and morbidity in patients with CHF. Nevertheless, the effects of VNS and beta-blockade could be different. As an example, previous studies indicated that VNS improves baroreflex neural arc in CHF rats [19] while metoprolol abolished dynamic sympathetic control of HR [20]. Moreover, beta-blockers can unmask an alpha-adrenergic vasoconstrictive effect, which can reduce coronary blood flow during sympathetic activation [21]. These mechanisms may partly contribute to the different outcomes between SSM and VSM.

In the present study, we created permanent MI and started treatments 2 weeks after MI. At this stage, MI might have been fixed, resulting in no significant difference in the MI size among the three groups. Because the observation period was relatively short (50 days), there was less edema, which resulted in no differences in body weight, lung weight, or liver weight among the three groups. SSM treatment decreased plasma epinephrine and BNP levels, which may partially contribute to the improvement in cardiac function. In patients with $\mathrm{CHF}$, compensatory cardiac sympathetic activation that resulted from cardiac dysfunction is associated with poor prognosis [22]; plasma norepinephrine level is a univariate predictor of all-cause and cardiac mortality, and beta-blocker treatment correlates with lower mortality [23]. However, the VSM group not only had significantly lower heart weight than the SS and SSM groups, but also prevented cardiac dysfunction, and decreased norepinephrine and BNP levels more than the SSM. These results suggest that VSM may decrease sympathetic outflow and improve neurohumoral state, and then effectively prevent cardiac remodeling. For the survival rate, although there was no difference between VSM and SSM groups (92 vs. 73\%, $P=0.163$ ), VSM markedly improved the 50-day survival (92 vs. $61 \%, P=0.022$ ) compared with the SS group.

Meanwhile, when we adopted data from our previous study [9], VSM therapy provided the similar treatment effects to that attained by VNS alone (normalized biventricular weight: $2.67 \pm 0.08$ vs. $2.75 \pm 0.08$; LVEDP: $16 \pm 1$ vs. $17 \pm 2 ; \mathrm{LV}+\mathrm{d} p / \mathrm{d} t_{\max }: 5036 \pm 172$ vs. $4152 \pm 75 \mathrm{mmHg} / \mathrm{s}$ ), and from the viewpoint of 50-day survival, 92 vs. $91 \%$ in CHF rats. These results indicated that the effect of VNS was not enhanced by metoprolol, i.e., there would be no significant synergistic effect between VNS and beta-blocker therapy. The difference between VSM and SSM may be attributable to mechanisms unique to VNS independent of ant-beta-adrenergic mechanism.

\section{Possible treatment mechanisms involved in VNS therapy}

The vagal nerve is an ancient nerve in evolution; it is composed of afferent and efferent fibers to govern many visceral 
reflexes. In small animals, such as mice, rats, and rabbits, the vagal nerve in the cervical region can be surgically separated from other nerves (e.g., aortic nerve and sympathetic nerve). Because various nerve fibers in the vagal nerve have different trigger levels to evoke action potential, responses to VNS rely on stimulus strength. Early acute VNS intervention for cardiac protection is considered as vagal efferent effect [24]. However, reviewing over 10 years of research on VNS for CHF including experimental and clinical studies, it may be worth focusing on the role of the vagal afferent pathway in the VNS therapy. We applied telemetry recording and remote controlling systems to evaluate behavioral and hemodynamic responses to VNS in free-moving rats. In the preliminary test for selecting VNS intensity, we observed that animals could sense the VNS even at very low intensity (current) and make behavioral responses such as holding breath and displaying alertness. These phenomena suggest that at least a part of the vagal afferent fibers can be stimulated by a low current, which may be lower than the trigger level of efferent fibers to control HR [25]. The titration for chronic VNS had to be set below a tolerable level in an experimental study [26] or a clinical trial [27].

At least three aspects of vagal afferent action should be considered. First, vagal afferent stimulation may inhibit sympathetic nerve activity through the input to the brainstem (the nucleus tractus solitarius) [28, 29]. This effect is possibly one of the most important mechanisms of VNS benefits for CHF, because persistent compensatory sympathetic activation for cardiac dysfunction leads to an increased secretion of neurohumoral factors such as vasopressin and adrenocorticoid. This increased secretion, in turn, further activates compensatory mechanisms to form a vicious cycle. We also observed that VNS treatment significantly improved the ability of the carotid sinus baroreflex to reduce sympathetic nerve activity in rats with CHF [19]. Second, electrical VNS decreases the release of various cytokines including tumor necrosis factor-alpha (TNF- $\alpha$ ) and interleukin- 6 (IL-6). This effect involves both afferent and efferent fibers and is called the cholinergic anti-inflammatory reflex [30]. Although this study did not evaluate these inflammatory factors, a previous study indicated that VNS in the anesthetized rodent following myocardial ischemia and reperfusion reduced infarct size, through the alpha-7 nicotinic acetylcholine receptordependent anti-inflammation mechanism [31]. In the canine model of CHF, both TNF- $\alpha$ and IL- 6 in plasma and in cardiac tissue are elevated more than in normal dogs, and they were attenuated by long-term monotherapy with VNS [32]. Third, vagal afferent stimulation may inhibit arginine vasopressin (AVP) secretion. This field rarely has attracted the attention of researchers in VNS therapy studies for patients with CHF. AVP is the most important neurohormone to maintain circulatory volume and fluid balance through regulation of water reabsorption in the kidney. Early studies have proven that the vagal afferent nerves exert a significant tonic inhibitory influence on AVP secretion in anesthetic and conscious dogs [33, 34]. AVP is reportedly upregulated and exhibits a defective response to physiological stimulation in CHF $[35,36]$. Although AVP was not measured in the present study, we have previously demonstrated that chronic VNS decreases AVP secretion and sodium ingestion in CHF rats with MI [16]. Therefore, we postulate that decreasing the AVP secretion may be a contributing mechanism of VNS therapy for CHF.

\section{Clinical implications}

The diversity of disease conditions in patients with CHF is one of the biggest challenges in clinical studies. According to therapeutic ethics, numerous clinical studies had to be performed in the presence of a routine therapeutic background. These therapeutic factors may obscure the real effects of the intervention to be evaluated. All the VNS clinical trials were conducted with the standard treatment background including beta-blockers, ACEI or/and ARB, aldosterone antagonists, and diuretic agents. In particular, most of the patients (92-100\%) used beta-blockers. The results of this study showed that VNS did not further decrease mean HR under the beta-blocker treatment background while it exerted additional benefits for CHF. Hence, beta-blockers should not be a malefactor of obstructing the VNS benefits in clinical trials. In a previous study, we demonstrated that pharmacological vagal activation by donepezil (an acetylcholinesterase inhibitor) combined with losartan exerts synergic treatment effects in rats with CHF [17]. Therefore, ACEI or ARB may be excluded from a malefactor list. We suspect that diuretic agents could interfere with the VNS effects. Clinical studies reported that diuretics increase sympathetic outflow and AVP secretion, without improving the long-term outcome in patients with CHF [37-41]. Diuretics may antagonize the above-mentioned VNS-induced prevention of AVP secretion. However, detailed data analysis to evaluate the VNS effects in diuretic-free patients with $\mathrm{CHF}$ is still lacking. The ongoing clinical trial ANTHEM-HFpEF has been designed to conduct VNS in patients with CHF with preserved and mid-range left ventricular ejection fraction [42]. Patients in these categories were less frequently prescribed diuretic agents than in subjects with severe CHF enrolled in previous VNS trials. We hope this prospective clinical study may provide new insights into diuretic influence on VNS therapy.

\section{Study limitations}

The present study has several limitations. First, it applied VNS to a rat model of MI-induced CHF. These rats were in the early stage of progression of cardiac remodeling and further development to CHF. However, the patients enrolled 
in clinical trials were usually in the late stage of CHF. Therefore, the therapeutic effects may be different and associated with different outcomes. Second, rodents and humans have different vagal nerve anatomy in the cervical area, which may cause varied functional effects of VNS. Third, they greatly differ in breath rate and HR. Finally, titration of VNS should be adjusted according to the response of the patients. For example, a VNS frequency of $20 \mathrm{~Hz}$ had been selected for the rat, which may not be the best frequency for humans. The new ongoing ANTHEM-HFpEF trial using a frequency of $5-10 \mathrm{~Hz}$ (near the natural discharge frequency of vagal) [42] is the new challenge.

\section{Conclusions}

VSM therapy further prevented cardiac remodeling, preserved cardiac output, and improved 50-day survival rate despite the background of beta-blockade in rats with CHF. The results suggest that VNS may exert its beneficial effects on the failing heart independently of its anti-beta-adrenergic mechanism. The VNS therapy may be applied to patients with $\mathrm{CHF}$ taking beta-blockers.

Acknowledgements This study was partly supported by JSPS KAKENHI (Grant Number: C-26461099, 17K09544).

Author contributions ML, CZ, and MS designed the study. ML and $\mathrm{CZ}$ performed the measurements and statistical analysis, and drafted the manuscript. KT, MI, and KU joined in interpreting the data. All authors have read and approved the final manuscript.

\section{Compliance with ethical standards}

Conflict of interest The author(s) declare that they have no competing interests.

Research involving animals The care of animals and all animal experiments were performed in strict accordance with the Guide for the Care and Use of Laboratory Animals published by the US National Institutes of Health (NIH Publication No. 85-23, revised 1996), and the Guiding Principles for the Care and Use of Animals in the Field of Physiological Sciences, which have been approved by the Physiological Society of Japan. All protocols were reviewed and approved by the Animal Subject Committee in the National Cerebral and Cardiovascular Center.

\section{References}

1. Pfeffer MA (1995) Left ventricular remodeling after acute myocardial infarction. Annu Rev Med 46:455-466

2. Hammermeister KE, DeRouen TA, Dodge HT (1979) Variables predictive of survival in patients with coronary disease. Selection by univariate and multivariate analyses from the clinical, electrocardiographic, exercise, arteriographic, and quantitative angiographic evaluations. Circulation 59:421-430
3. Likoff MJ, Chandler SL, Kay HR (1987) Clinical determinants of mortality in chronic congestive heart failure secondary to idiopathic dilated or to ischemic cardiomyopathy. Am J Cardiol 59:634-638

4. Vaughan DE, Pfeffer MA (1994) Angiotensin converting enzyme inhibitors and cardiovascular remodelling. Cardiovasc Res 28:159-165

5. Packer M, Bristow MR, Cohn JN, Colucci WS, Fowler MB, Gilbert EM, Shusterman NH (1996) The effect of carvedilol on morbidity and mortality in patients with chronic heart failure. U.S. Carvedilol Heart Failure Study Group. N Engl J Med 334:1349-1355

6. Packer M, Coats AJ, Fowler MB, Katus HA, Krum H, Mohacsi P, Rouleau JL, Tendera M, Castaigne A, Roecker EB, Schultz MK, DeMets DL (2001) Effect of carvedilol on survival in severe chronic heart failure. N Engl J Med 344:1651-1658

7. La Rovere MT, Bigger JT Jr, Marcus FI, Mortara A, Schwartz PJ (1998) Baroreflex sensitivity and heart-rate variability in prediction of total cardiac mortality after myocardial infarction. ATRAMI (Autonomic Tone and Reflexes After Myocardial Infarction) Investigators. Lancet 351:478-484

8. Lechat P, Hulot JS, Escolano S, Mallet A, Leizorovicz A, Werhlen-Grandjean M, Pochmalicki G, Dargie H (2001) Heart rate and cardiac rhythm relationships with bisoprolol benefit in chronic heart failure in CIBIS II trial. Circulation 103:1428-1433

9. Li M, Zheng C, Sato T, Kawada T, Sugimachi M, Sunagawa K (2004) Vagal nerve stimulation markedly improves long-term survival after chronic heart failure in rats. Circulation 109:120-124

10. De Ferrari GM, Stolen C, Tuinenburg AE, Wright DJ, Brugada J, Butter C, Klein H, Neuzil P, Botman C, Castel MA, D'Onofrio A, de Borst GJ, Solomon S, Stein KM, Schubert B, Stalsberg K, Wold N, Ruble S, Zannad F (2017) Long-term vagal stimulation for heart failure: eighteen month results from the NEural Cardiac TherApy foR Heart Failure (NECTAR-HF) trial. Int J Cardiol 244:229-234

11. Gold MR, Van Veldhuisen DJ, Hauptman PJ, Borggrefe M, Kubo SH, Lieberman RA, Milasinovic G, Berman BJ, Djordjevic S, Neelagaru S, Schwartz PJ, Starling RC, Mann DL (2016) Vagus nerve stimulation for the treatment of heart failure: the INOVATEHF trial. J Am Coll Cardiol 68:149-158

12. Premchand RK, Sharma K, Mittal S, Monteiro R, Dixit S, Libbus I, DiCarlo LA, Ardell JL, Rector TS, Amurthur B, KenKnight BH, Anand IS (2014) Autonomic regulation therapy via left or right cervical vagus nerve stimulation in patients with chronic heart failure: results of the ANTHEM-HF trial. J Card Fail 20:808-816

13. Gronda E, Vanoli E (2016) Autonomic modulation with baroreflex activation therapy in heart failure. Curr Heart Fail Rep $13: 273-280$

14. Sabbah HN, Imai M, Zaretsky A, Rastogi S, Wang M, Jiang A, Zaca V (2007) 509 Therapy with vagus nerve electrical stimulation combined with beta-blockade improves left ventricular systolic function in dogs with heart failure beyond that seen with beta-blockade alone. European Journal of Heart Failure Supplements 6:114

15. Li M, Zheng C, Kawada T, Inagaki M, Uemura K, Shishido T, Sugimachi M (2013) Donepezil markedly improves long-term survival in rats with chronic heart failure after extensive myocardial infarction. Circ J 77:2519-2525

16. Li M, Zheng C, Inagaki M, Kawada T, Sunagawa K, Sugimachi M (2005) Chronic vagal stimulation decreased vasopressin secretion and sodium ingestion in heart failure rats after myocardial infarction. Conf Proc IEEE Eng Med Biol Soc 4:3962-3965

17. Li M, Zheng C, Kawada T, Inagaki M, Uemura K, Sugimachi M (2014) Adding the acetylcholinesterase inhibitor, donepezil, to losartan treatment markedly improves long-term survival in rats with chronic heart failure. Eur J Heart Fail 16:1056-1065 
18. Komajda M, Isnard R, Cohen-Solal A, Metra M, Pieske B, Ponikowski P, Voors AA, Dominjon F, Henon-Goburdhun C, Pannaux M, Bohm M, prEserve DlvefchFwisI (2017) Effect of ivabradine in patients with heart failure with preserved ejection fraction: the EDIFY randomized placebo-controlled trial. Eur J Heart Fail 19:1495-1503

19. Kawada T, Li M, Zheng C, Shimizu S, Uemura K, Turner MJ, Yamamoto H, Sugimachi M (2014) Chronic vagal nerve stimulation improves baroreflex neural arc function in heart failure rats. J Appl Physiol (1985) 116:1308-1314

20. Kawada T, Shimizu S, Uemura K, Hayama Y, Yamamoto H, Shishido T, Nishikawa T, Sugimachi M (2018) Ivabradine preserves dynamic sympathetic control of heart rate despite inducing significant bradycardia in rats. J Physiol Sci. https://doi. org/10.1007/s12576-018-0636-2

21. Guth BD, Heusch G, Seitelberger R, Ross J Jr (1987) Mechanism of beneficial effect of beta-adrenergic blockade on exercise-induced myocardial ischemia in conscious dogs. Circ Res 60:738-746

22. Vaseghi M, Shivkumar K (2008) The role of the autonomic nervous system in sudden cardiac death. Prog Cardiovasc Dis 50:404-419

23. Cohen-Solal A, Jacobson AF, Pina IL (2017) Beta blocker dose and markers of sympathetic activation in heart failure patients: interrelationships and prognostic significance. ESC Heart Fail 4:499-506

24. Vanoli E, De Ferrari GM, Stramba-Badiale M, Hull SS Jr, Foreman RD, Schwartz PJ (1991) Vagal stimulation and prevention of sudden death in conscious dogs with a healed myocardial infarction. Circ Res 68:1471-1481

25. Qing KY, Wasilczuk KM, Ward MP, Phillips EH, Vlachos PP, Goergen CJ, Irazoqui PP (2018) B fibers are the best predictors of cardiac activity during vagus nerve stimulation. Bioelectron Med $4: 5$

26. Nishizaki A, Sakamoto K, Saku K, Hosokawa K, Sakamoto T, Oga Y, Akashi T, Murayama Y, Kishi T, Ide T, Sunagawa K (2016) Optimal titration is important to maximize the beneficial effects of vagal nerve stimulation in chronic heart failure. J Card Fail 22:631-638

27. Schwartz PJ, De Ferrari GM, Sanzo A, Landolina M, Rordorf R, Raineri C, Campana C, Revera M, Ajmone-Marsan N, Tavazzi L, Odero A (2008) Long-term vagal stimulation in patients with advanced heart failure: first experience in man. Eur J Heart Fail 10:884-891

28. Saku K, Kishi T, Sakamoto K, Hosokawa K, Sakamoto T, Murayama Y, Kakino T, Ikeda M, Ide T, Sunagawa K (2014) Afferent vagal nerve stimulation resets baroreflex neural arc and inhibits sympathetic nerve activity. Physiol Rep 2:e12136

29. Smith BN, Dou P, Barber WD, Dudek FE (1998) Vagally evoked synaptic currents in the immature rat nucleus tractus solitarii in an intact in vitro preparation. J Physiol 512(Pt 1):149-162
30. Tracey KJ (2002) The inflammatory reflex. Nature 420:853-859

31. Kiss A, Tratsiakovich Y, Mahdi A, Yang J, Gonon AT, Podesser BK, Pernow J (2017) Vagal nerve stimulation reduces infarct size via a mechanism involving the alpha-7 nicotinic acetylcholine receptor and downregulation of cardiac and vascular arginase. Acta Physiol (Oxf) 221:174-181

32. Hamann JJ, Ruble SB, Stolen C, Wang M, Gupta RC, Rastogi S, Sabbah HN (2013) Vagus nerve stimulation improves left ventricular function in a canine model of chronic heart failure. Eur J Heart Fail 15:1319-1326

33. Morita H, Manders WT, Skelton MM, Cowley AW Jr, Vatner SF (1986) Vagal regulation of arginine vasopressin in conscious dogs. Am J Physiol 251:H19-H23

34. Thames MD, Schmid PG (1981) Interaction between carotid and cardiopulmonary baroreflexes in control of plasma ADH. Am J Physiol 241:H431-H434

35. Goldsmith SR (1992) Baroreflex loading maneuvers do not suppress increased plasma arginine vasopressin in patients with congestive heart failure. J Am Coll Cardiol 19:1180-1184

36. Goldsmith SR, Francis GS, Levine TB, Cowley JAW, Cohn JN (1983) Impaired response of plasma vasopressin to orthostatic stress in patients with congestive heart failure. J Am Coll Cardiol 2:1080-1083

37. Wu MY, Chang NC, Su CL, Hsu YH, Chen TW, Lin YF, Wu CH, Tam KW (2014) Loop diuretic strategies in patients with acute decompensated heart failure: a meta-analysis of randomized controlled trials. J Crit Care 29:2-9

38. Felker GM, O'Connor CM, Braunwald E, Heart Failure Clinical Research Network I (2009) Loop diuretics in acute decompensated heart failure: necessary? Evil? A necessary evil? Circ Heart Fail 2:56-62

39. Konstam MA, Gheorghiade M, Burnett JC Jr, Grinfeld L, Maggioni AP, Swedberg K, Udelson JE, Zannad F, Cook T, Ouyang J, Zimmer C, Orlandi C, Efficacy of Vasopressin Antagonism in Heart Failure Outcome Study With Tolvaptan I (2007) Effects of oral tolvaptan in patients hospitalized for worsening heart failure: the EVEREST Outcome Trial. JAMA 297:1319-1331

40. Bayliss J, Norell M, Canepa-Anson R, Sutton G, Poole-Wilson P (1987) Untreated heart failure: clinical and neuroendocrine effects of introducing diuretics. Br Heart J 57:17-22

41. Francis GS, Siegel RM, Goldsmith SR, Olivari MT, Levine TB, Cohn JN (1985) Acute vasoconstrictor response to intravenous furosemide in patients with chronic congestive heart failure. Activation of the neurohumoral axis. Ann Intern Med 103:1-6

42. DiCarlo LA, Libbus I, Kumar HU, Mittal S, Premchand RK, Amurthur B, KenKnight BH, Ardell JL, Anand IS (2018) Autonomic regulation therapy to enhance myocardial function in heart failure patients: the ANTHEM-HFpEF study. ESC Heart Fail 5:95-100 